\title{
Perioperative Dental Injuries: A Retrospective Analysis of Documented Injuries at Penn State Hershey Medical Center
}

\author{
Sarah H. Nie, Sonia J. Vaida, Jansie Prozesky \\ Department of Anesthesiology, Penn State Hershey Medical Center, Hershey, USA \\ Email: jprozesky@hmc.psu.edu
}

Received 29 January 2015; accepted 13 April 2015; published 15 April 2015

Copyright (C) 2015 by authors and Scientific Research Publishing Inc.

This work is licensed under the Creative Commons Attribution International License (CC BY). http://creativecommons.org/licenses/by/4.0/

(c) (i) Open Access

\begin{abstract}
Background: Dental injury is the most common cause of malpractice claims against anesthesiologists in the United States. This study analyzed the incidence, risk factors, and consequences of dental injury directly attributed to anesthetic care at the Penn State Hershey Medical Center. Methods: Data was collected from retrospective chart review of available dental injury records, incident reports, and filed dental claims from January 2008 to June 2014. Results: Forty seven dental injuries were documented amongst 247,323 general anesthetics representing an overall incidence of $0.019 \%$. Seventy percent of injuries occurred in patients with documented pre-existing prosthodontics or poor dentition and largely involved maxillary teeth $(68 \%)$. Dental fracture $(40 \%)$ and avulsion (40\%) were the most common types of injury. Direct laryngoscopy was used in $60 \%$ of cases reporting dental injury; more than one attempt at securing the airway was required in $36 \%$ of cases. Total costs related to the injuries were \$24347.65. Conclusions: Dental injury is often attributed to the actions of the anesthesiologist. The injury may be unnoticed until after surgery, what makes it difficult to identify the time and mechanism of injury. Our results suggest that the number of attempts at securing an airway is a risk factor for dental injury. Proper preoperative documentation of poor dentition, discussion with the patient about the increased risk of dental injury and detailed documentation of dental injury when it occurs is advised.
\end{abstract}

\section{Keywords}

General Anesthesia, Dental Injury 


\section{Introduction}

Dental injury is the most common malpractice claim against anesthesiologists, comprising approximately one third of all medicolegal claims in the United States [1]. The costs and morbidity related to dental injuries are significant. The reported incidence of perioperative dental injury varies from $0.02 \%$ to $0.04 \%$ in retrospective studies. [2]-[4] Poor dentition, reconstructive work, and difficult intubation were described as risk factors [2]-[6]. The teeth most likely to be injured are the upper incisors [2]-[6]. Newland et al., [2] found that $86 \%$ of dental injuries were discovered by the anesthesiologist, whereas $14 \%$ were missed and reported by the patient.

The purpose of our study was to analyze the incidence and identify risk factors and consequences of dental injury directly attributed to the anesthetic management at Penn State Hershey Medical Center (PSHMC) to improve quality of care.

\section{Materials and Methods}

After obtaining approval from the PSHMC Institutional Review Board, case incidences were compiled from filed dental claims, paper post-operative anesthesia care unit (PACU) incident reports, intraoperative electronic medical records (EMRs), and electronic post-operative assessments from January 2008 to June 2014. The workforce in the department consisted of 48 attendings, 68 residents, 20 CRNAs. After identifying the cases, data were obtained from the post-operative hospital course and anesthesia records, pre-operative anesthesia clinic visit, dentist consultation record, dental itemized billing statement, and any documented phone messages or other correspondences.

Our institution, like many, was undergoing a transition period from paper to EMRs in July 2011. Dental injury was a distinct category, under "complications", that was later added to our EMR. It is required that a complication, or lack thereof, be noted in the anesthetic record, by the primary anesthesia provider and the PACU anesthesiology resident.

Dental injury was defined as any change to a patient's dentition, during the perioperative period, attributed to an anesthesiologist providing direct care. Pre-existing dental condition were defined as decayed or restored teeth; e.g., periodontal disease, poor dentition, or dental prosthetics. A few illustrative excluded cases include: accidentally dropped dentures by an anesthesiology resident in a pre-operative area (compensation \$86), displaced tooth upon endoscope placement by a surgeon, multiple incidences of avulsion of tooth with endoscopy bite block, and lost dentures at the end of a complicated hospitalization with multiple trips to the operating room (compensation \$1600 split between Anesthesia Department and Cardiothoracic Department).

Injuries were categorized as: "fractures", defined as damage to the enamel; "subluxation or luxation", defined as damage to tooth supporting structures resulting in abnormal loosening without displacement in the former or partial displacement in the latter; and "avulsion", defined as total displacement [2].

Clinical and demographic data gathered included incidence of dental injury, patient characteristics (age, weight, height, BMI, gender, ASA status, Malampati score, pre-existing periodontal disease, dental prosthetics), level of training of provider, timing of injury, intubation technique used, specific tooth/teeth injured, follow up/ therapy of dental injuries, and cost incurred by the department for repair.

\section{Results}

\subsection{Incidence and Patient Demographics}

A total of 47 dental injuries were documented amongst 247,323 general anesthetics, representing an overall incidence of $0.019 \%$. Occurrence of dental injury by time period is shown in Table 1 . Patient demographics are shown in Table 2. Ages ranged from 4 to 83 years old, with a mean of $46.8 \pm 23$. The mean BMI was $27.6 \pm 8$. All but two patients were ASA status II or III. Three cases were emergent. Sixty-six percent of the patients had Mallampati scores of 1 or 2 (Table 2). Seventy percent of patients $(n=33)$ had documented pre-existing prosthodontics or poor dentition.

\subsection{Distribution and Modality of Injury}

In $81 \%$ of cases, only one tooth was affected $(n=38)$, and in $9 \%$ of cases two teeth were affected $(n=4)$. In 5 cases the injury was not specifically documented. Thus, from all cases where dental injury was specifically do- 
Table 1. Incidence of anesthesiologist-related dental injuries by year.

\begin{tabular}{|c|c|c|c|c|c|c|c|}
\hline Year & $\begin{array}{c}1 / 2008- \\
6 / 2008\end{array}$ & $\begin{array}{c}7 / 2008- \\
6 / 2009\end{array}$ & $\begin{array}{c}\text { 7/2009- } \\
6 / 2010\end{array}$ & $\begin{array}{c}\text { 7/2010- } \\
6 / 2011\end{array}$ & $\begin{array}{c}\text { 7/2011- } \\
6 / 2012\end{array}$ & $\begin{array}{c}07 / 2012- \\
06 / 2013\end{array}$ & $\begin{array}{c}07 / 2013- \\
06 / 2014\end{array}$ \\
\hline Total Anesthetics & 16,989 & 34,603 & 34,847 & 38,017 & 41,130 & 39,852 & 41,885 \\
\hline Number of Dental Injuries & 3 & 8 & 3 & 1 & 6 & 14 & 12 \\
\hline Incidence \% & $0.018 \%$ & $0.023 \%$ & $0.008 \%$ & $0.002 \%$ & $0.015 \%$ & $0.035 \%$ & $0.029 \%$ \\
\hline
\end{tabular}

Table 2. Patients' demographic data.

\begin{tabular}{cccc}
\hline Characteristics & & Number (n) & Percentage (\%) \\
\hline Gender & Male & 28 & 59.6 \\
& Female & 19 & 40.4 \\
ASA & & & 2.1 \\
& I & 1 & 46.8 \\
& II & 22 & 40.4 \\
III & IV & 19 & 6.4 \\
Mallampati Score & V & 3 & 0 \\
& E & 0 & 6.4 \\
& Missing Documentation & 3 & 4.2 \\
\hline & I & 2 & 27.7 \\
\hline
\end{tabular}

cumented, 42 injuries to teeth were noted, with the majority of those involving maxillary teeth $(78 \%, \mathrm{n}=32)$ Central incisors (\#8 and 9) were the mandibular teeth most frequently affected (47\%) (Figure 1). The modalities of the documented dental injuries are presented in Table 3.

\subsection{Timing of Injury and Airway Management}

Forty two percent of dental injuries $(n=20)$ occurred upon intubation, $11 \%(n=5)$ upon extubation, and in $47 \%$ $(n=22)$, the timing of the injury was not documented or not specifically known, such as when a tooth was found in the mouth at extubation but intubation was documented as atraumatic. Direct laryngoscopy was used 60\% of the time $(n=28)$, laryngeal mask airway in 28\% $(n=13)$, Glidescope in $11 \%(n=5)$ and fiberoptic intubation in $2 \%(n=1)$. One attempt at securing the airway was made in $55 \%$ of cases $(n=26)$, two attempts in $17 \%(n=8)$, three attempts in $13 \%(n=6)$, and four attempts in $2 \%(n=1)$. The number of attempts were not documented in $13 \%$ of cases $(n=6)$.

\subsection{Anesthesia Provider Level of Training}

Details, of the level of training of the anesthesia providers, are presented in Table 4. The attending was usually the second person to attempt intubation after a failed attempt by the resident or certified nurse anesthetist. 


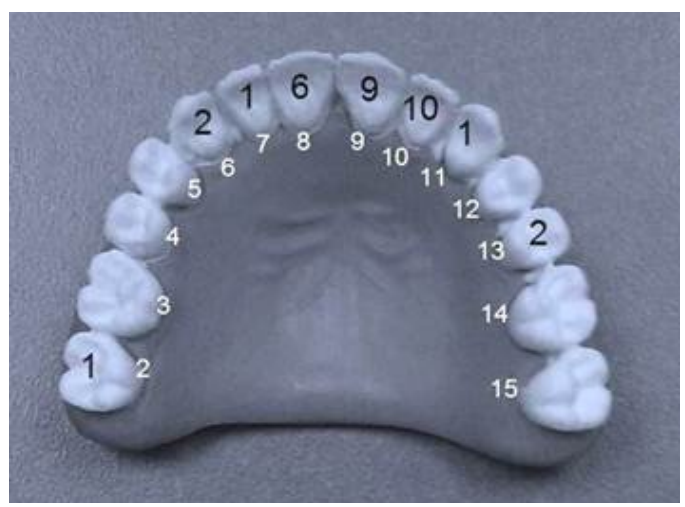

Figure 1. Incidence of injury to mandibular teeth. The white numbers indicate common numbering of mandibular teeth and the black numbers indicate the number of injuries, noted in our study, to have occurred to that tooth.

Table 3. Modality of dental injury.

\begin{tabular}{lc}
\hline Type of Injury & Number of Occurrences \\
\hline Fracture & 17 \\
Avulsion & 17 \\
Subluxation & 6 \\
Damaged Bridge & 2 \\
Others & 2 \\
Missing Documentation & 5 \\
\hline
\end{tabular}

Table 4. Anesthesia provider level of training.

\begin{tabular}{lc}
\hline \multicolumn{1}{c}{ Level of Training } & Number of Dental Injuries \\
\hline $\begin{array}{l}\text { Attending anesthesiologist } \\
\text { Certified registered nurse } \\
\text { anesthetist }\end{array}$ & 11 \\
Resident postgraduate year 1 & 7 \\
Resident postgraduate year 2 & 11 \\
Resident postgraduate year 3 & 9 \\
Resident postgraduate year 4 & 14 \\
\hline
\end{tabular}

\subsection{Documentation}

Out of 47 cases, 9 had no documentation in the medical record of dental injuries and were noted only when the patient requested monetary compensation. There was one case where the intraoperative paper anesthetic record could not be obtained. In 8 cases, the dental injury was discovered postoperatively in the PACU.

\subsection{Follow up Therapy and Cost}

Forty-four percent of the patients with dental injury had documented follow up, either via examination, therapy by dentist, or in the PACU, such as via chest X-ray to check for aspiration $(n=21)$. Twenty patients $(42 \%)$ received payment. Twenty-five patients had no known therapy for their injuries, and of these, 2 cases had a com- 
pensated dental exam but decided on no therapy. Cost was not calculated for the following cases: one case of bedside dental extraction by the dentist on call, four cases of post-operative chest X-rays to identify missing dentition, one case of an ENT consult for intra-operative bronchoscopy, and one case of an additional endoscopy procedure to retrieve a swallowed bridge. A total of 3 dental extractions, 4 surface restorations, 6 crowns, 8 prosthetic repairs or creation of a new prosthetic, and 3 root canals were performed. The total cost to the PSHMC Anesthesia department was \$24347.65 (range: \$33 to \$5255, median: \$530).

\section{Discussion}

The cost of dental injury is more than monetary. Although it is a well-known risk of anesthetic care, dental injury can cause unexpected distress to patients, and not just of a cosmetic nature. Significant potential morbidity can occur, such as with an undetected loose tooth in the airway. For example, at our institution, a patient had to undergo a second procedure and exposure to anesthesia to retrieve a swallowed dental bridge discovered on abdominal X-ray after the patient reported it missing in the PACU.

The incidence of known dental injury at PSHMC was determined to be $0.019 \%$, representing 1:5262 cases, which compares favorably to incidences of $0.02 \%$ to $0.04 \%$ reported from other retrospective studies [2] [3]. Chen et al., [7] found an incidence of up to $12.1 \%$ in his prospective study. The wide range of incidences reflects upon patient population and study design. Lockhart et al., [8] surveyed 133 anesthesiology training programs and found an average incidence of $0.1 \%$ for dental injury during endotracheal intubations. This was corroborated with a study by Gaiser and colleagues, who found a similar $0.1 \%$ incidence of injury involving anesthesia residents in training [9]. However, other university training institutions have quoted an incidence rate closer to $0.02 \%-0.04 \%$ [2]-[4].

The increasing incidence of dental injury over time can be explained by improved documentation and reporting due to electronically captured data through the EMR.

Due to the retrospective nature of our study, there were shortcomings in capturing all incidences of dental injury and incomplete record keeping. There are likely unrecorded, unclaimed incidences, and lost paper records. Furthermore, there is, unfortunately, no feasible way to collect incidences that occurred before the implementation of our EMR and that were missed by examining formal claims for reimbursement and paper PACU incident reports.

The typical patient sustaining dental injury, according to our findings, was older than 50 years, had a median BMI of 29, was ASA II or III, non-emergent, and with a Mallampati score of 2 . Approximately $40 \%$ of the injuries occurred when more than one attempt at direct laryngoscopy was performed. Although our study does not have a control group, these findings would suggest that, in at least some cases, the anesthesiologist encountered a more difficult airway than anticipated. This is supported by studies that have shown that there was an assessment of an anticipated difficult airway in only $19 \%$ - 35\% of dental injury cases [2] [6].

Tracheal intubation, a difficult-to-intubate airway, and poor dentition have been the only significant risk factors reported in the literature [2] [4]. Surprisingly, obesity, rheumatoid arthritis, and history of head/neck cancer have not been shown to be statistically significant [2] [4]. Newland, et al., found patients with poor dentition or reconstructive work, and whose tracheas were difficult to intubate, had a 20-fold higher risk of dental injury. Among those easy to intubate, poor dentition or reconstructive work conferred a 3.4 times greater risk [2]. Warner, et al., found increased difficulty of intubation to increase risk by a factor of 11, and pre-existing poor dentition to increase risk by a factor of 50 [4]. In our study, 70\% of patients who experienced dental injury had a pre-existing dental condition. Like the authors of other studies [2]-[6], we found that the majority of teeth injured were the frontal incisors and fracture was the most common type of injury.

Dental injuries were most common during direct laryngoscopy with intubation. Our study also noted that a significant number of dental injuries, 28\%, occurred with use of the LMA, which is greater compared to the incidence (9.8\%) found by Adolphs, et al., and greater than that (1.3\%) found by Newland, et al. [2] [3]. Our finding that $11 \%$ of the dental injuries occurred upon extubation, is similar to other studies which have reported $8 \%-10 \%$ of dental injuries to occur upon extubation [3] [4]. In roughly half of the cases, the timing of injury was either unknown or not documented. This further emphasizes the need for vigilance since dental injury can occur in a wide variety of unexpected circumstances.

Out of 47 cases, 9 had no documentation of dental injury in the medical record and were noted only because the patient requested compensation. In 8 cases, the injury was discovered post-operatively in the PACU by the 
patient. Therefore, $36 \%$ of the time, the primary provider was unaware that a dental injury had occurred. In the study by Newland et al., $86 \%$ of injuries were initially discovered by the provider and only $14 \%$ by the patient. However, our study design identified many cases from the billing department as opposed to a database of adverse events only.

Among those who filed for monetary compensation, 45\% (9 out of 20) had no documentation from either the anesthetic record/primary anesthesia provider or in the post anesthesia care unit. It may be that either documentation was not completed at the time or that the damage was discovered by the patient after discharge. There is the possibility of malingering where no dental injury occurred.

Our findings suggest that patients may be less likely to request monetary compensation if their injury is recognized at the time of their care. Twenty seven of 37 patients who did have documentation, by either the primary anesthesia provider or PACU personnel, did not file for monetary compensation after discharge. Probably, a physician had talked with the patient about their injury.

\section{Conclusion}

In summary, at our institution we found that dental injuries are often unrecognized or undocumented by the primary anesthesia provider. Our results also suggest that the number of attempts at intubation may be a significant risk factor for dental injury. Greater awareness and detection of dental injuries, as well as discussion of the injury with the patient is important. Documentation of poor dentition and injury when it occurs is strongly advised. As a result of this study we increased the education and awareness of the departmental in preventing dental injuries.

\section{References}

[1] Cass, N.M. (2004) Medicolegal Claims against Anaesthetists: A 20-Year Study. Anaesthesia and Intensive Care, 32, 47-58.

[2] Newland, M.C., Ellis, S.J., Peters, K.R., et al. (2007) Dental Injury Associated with Anesthesia: A Report of 161,687 Anesthetics Given over 14 Years. Journal of Clinical Anesthesia, 19, 339-345. http://dx.doi.org/10.1016/j.jclinane.2007.02.007

[3] Adolphs, N., Kessler, B., von Heymann, C., et al. (2011) Dentoalveolar Injury Related to General Anaesthesia: A 14 Years Review and a Statement from the Surgical Point of View Based on a Retrospective Analysis of the Documentation of a University Hospital. Dental Traumatology: Official Publication of International Association for Dental Traumatology, 27, 10-14.

[4] Warner, M.E., Benenfeld, S.M., Warner, M.A., Schroeder, D.R. and Maxson, P.M. (1999) Perianesthetic Dental Injuries: Frequency, Outcomes, and Risk Factors. Anesthesiology, 90, 1302-1305. http://dx.doi.org/10.1097/00000542-199905000-00013

[5] Yasny, J.S. (2009) Perioperative Dental Considerations for the Anesthesiologist. Anesthesia and Analgesia, 108, 15641573. http://dx.doi.org/10.1213/ane.0b013e31819d1db5

[6] Givol, N., Gershtansky, Y., Halamish-Shani, T., Taicher, S., Perel, A. and Segal, E. (2004) Perianesthetic Dental Injuries: Analysis of Incident Reports. Journal of Clinical Anesthesia, 16, 173-176. http://dx.doi.org/10.1016/j.jclinane.2003.06.004

[7] Chen, J.J., Susetio, L. and Chao, C.C. (1990) Oral Complications Associated with Endotracheal General Anesthesia. Anaesthesiologica Sinica, 28, 163-169.

[8] Lockhart, P.B., Feldbau, E.V., Gabel, R.A., Connolly, S.F. and Silversin, J.B. (1986) Dental Complications during and after Tracheal Intubation. Journal of the American Dental Association, 112, 480-483. http://dx.doi.org/10.14219/jada.archive.1986.0035

[9] Gaiser, R.R. and Castro, A.D. (1998) The Level of Anesthesia Resident Training Does Not Affect the Risk of Dental Injury. Anesthesia and Analgesia, 87, 255-257. http://dx.doi.org/10.1097/00000539-199808000-00004 